\title{
Nurturing Path of "Specialized, Elaborative, Characteristic" Little Giant Enterprises: Study of Southern Jiangsu Province
}

\author{
Zhang Rui, Shi Xiaopeng, Chen Yingwu
}

Jiangsu Institute of Economic and Information Technology, Nanjing 210003, China

\begin{abstract}
Accelerating the development of "specialized, elaborative, characteristic" little giant (SECLG) enterprises is essential in order to enhance the overall competitiveness of China's enterprises, encourage endogenous and innovation-driven growth, heighten total factor productivity, and implement strategic supply-side reforms. This is a necessary path that is adapted to China's national conditions and the sustained healthy development of the manufacturing industry. To discover such nurturing practices and experiences, the present paper focuses on southern Jiangsu Province, which is an important agglomeration area of China's SECLG enterprises. This paper may serve as a reference for SECLG enterprises nurturing policy making and may be useful in fields related to the in-depth implementation of an Innovation-driven Development Strategy and Industrial Strong Base Strategy.
\end{abstract}

Keywords: "specialized, elaborative, characteristic" little giant (SECLG); industrial base; policy system

\section{Introduction}

"Specialized, elaborative, characteristic" little giant (SECLG) enterprises are enterprises that focus on certain market segments, with leading international production technology and high market share. SECLGs are also known as single champion enterprises, "science and technology giant enterprises," "invisible champion enterprises," and "specialized, elaborative, characteristic and new" little giant enterprises. SECLGs constitute the cornerstone of innovation and development in the manufacturing industry. The implementation of cultivation and promotion action among SECLGs will help guide such enterprises in taking a long-term focus on areas of expertise and taking the road of specialized innovation. It will also facilitate the implementation of Made in China 2025, which concerns breakthrough key manufacturing areas, promote the manufacturing industry toward mid-to-high end products, and provide strong support for the realization of Industrial Strong Base Strategy and Manufacturing
Power Strategy. Additionally, it will help increase total factor productivity, enhance product added value, improve supply quality, foster the implementation of supply-side structural reform requirements, and ultimately facilitate the achievement of real improvement in social productivity.

\section{Literature review on SECLG enterprises}

German Herman Simon is the father of the "invisible champion." In 1986, he proposed the "invisible champion" theory. He believes that an "invisible champion" roughly meets three criteria: first, the company's product rankings cannot be less than the top three in the world market or first in the continental market; second, the annual turnover of the enterprise cannot exceed 5 billion euros; third, companies cannot be familiar with the average consumer. German export trade — and even overall economic development - mainly benefits from SMEs, especially a large number of "invisible champion" enterprises. According to this

Received date: September 15, 2017; revised date: September 25, 2017

Corresponding author: Zhang Rui, Jiangsu Institute of Economic and Information Technology, Department Director. Major research field is industrial economics. E-mail: james_zr@163.com

Funding program: CAE Advisory Project “Manufacturing Power Strategy" (2015-ZD-15) and “Industrial Strong Base Strategy” (2016-ZD-01)

Chinese version: Strategic Study of CAE 2017, 19(5): 097-102

Cited item: Zhang Rui et al. The Nurturing Path of "Specialized, Elaborative, Characteristic" Little Giant Enterprises: Study on Southern Jiangsu Province. Strategic Study of CAE, https://doi.org/10.15302/J-SSCAE-2017.05.017 
definition, there are currently a total of 2734 "invisible champion" enterprises in the world, of which 1307 are located in Germany, accounting for $47.8 \%$ of the total number. Of this number, 366 are located in the United States, which ranks second on the list, and only 68 are located in China, ranked eighth on the list [1] The rankings of countries in which "invisible champions" are located are shown in Table 1.

In recent years, domestic academics and political circles have begun to devote attention to the study of SECLG enterprises and have begun to discuss the related evaluation system, growth mode, and cultivation mode. Li Tianshu [2] argues that many problems, such as the low starting point, large number of industry barriers, and imperfect financing environment, require governments to improve the fiscal and tax support policies for science and technology SMEs, to establish public science and technology service platforms at all levels, to support venture capital investments, and to explore credit products suitable for science and technology SMEs. Sun Chunlan [3] suggests that improving the ability to innovate is a way for small and mediumsized technology-based enterprises to survive. It is important to strengthen the combination of production, learning, research, and cooperation between SMEs and to promote collaborative innovation among enterprises, research institutes, and universities. Zhao Huicun believes [4] that for the growth and upgrading of SECLG enterprises, the government must strengthen the "external brain" support, actively develop multi-level capital markets, and promote the integration of industrialization and informatization. Findings from existing research are relatively insufficient with respect to public policy theoretical research and path findings, have lacked systematic review of the guiding ideology, policy, and effect on the cultivation of small giant enterprises, and have lacked vivid case studies of advanced regions. We are currently implementing Industrial Strong Base Strategy and Manufacturing Power Strategy, it is important to take southern Jiangsu Province as an example to summarize the local experience of cultivating small giant enterprises in order to provide experiences for the whole nation.

\section{The main experience of nurturing SECLG enterprises in southern Jiangsu Province}

Southern Jiangsu Province includes Nanjing, Zhenjiang, Changzhou, Wuxi, and Suzhou. As one of the most developed economies in the Yangtze River Delta region, southern Jiangsu Province accounts for $0.29 \%$ of China's total land area but $6.1 \%$ of the total economy. Since the start of the reform and openingup, southern Jiangsu Province has been at the forefront of the entire nation and fostered a number of SECLG enterprises with prominent main businesses, strong competitiveness, and high growth rates, which has contributed in upgrading industry for the whole nation. The Jiangsu provincial government and the city governments have always attached great importance to the cultivation of SECLG enterprises. In November 2016, among the first list of manufacturing single champion enterprises in the country announced by the Ministry of Industry and Information Technology and the China Federation of Industrial Economics, there were 5 manufacturing demonstration single champions in southern Jiangsu Province (national number, 54), with 7 manufacturing nurturing single champions in southern Jiangsu Province (national number, 50).

The targeted nurturing of SECLG enterprises in southern Jiangsu Province can be roughly traced back to 2006. At that time, the booming industrial development created strong economic upgrade demand. Policies at the provincial and city level started to encourage industrial innovation and R\&D. Considering the issuing of Special Instructions on Cultivating 10000 SECLG Enterprises in Jiangsu Province [5] a separation node, the nurturing of SECLG enterprises can be divided into two stages. The stage before 2012 can be defined as the production factor support period, and the stage after 2012 can be defined as the systematic promotion of SECLG cultivation period. The standard of SECLGs in Jiangsu Province has also been constantly improving. The 2017 version includes five main aspects. First, company R\&D costs accounted for more than $3 \%$ of sales revenue. Second, there was more than 50 million yuan of main business revenue or more

Table 1. "Invisible champion" country rankings.

\begin{tabular}{lcc}
\hline Ranking & Country & Number of "invisible champions" \\
\hline 1 & Germany & 1307 \\
2 & USA & 366 \\
3 & Japan & 220 \\
4 & Austria & 116 \\
5 & Switzerland & 110 \\
6 & Italy & 76 \\
7 & France & 75 \\
8 & China & 68 \\
9 & UK & 67 \\
\hline
\end{tabular}


than 8 million yuan of profit tax in the previous year. Third, the main product market share was among the top five in the domestic market. Fourth, the operating income average growth rate has been $10 \%$ or more in the past three years. Finally, the enterprises are managed properly with no substantial dishonesty.

\subsection{First stage: provide production factors for SECLGs}

At this stage, there are few enterprises in southern Jiangsu Province that meet the standards of SECLGs, and many SMEs remain in the process of transforming from an extensive development mode to an intensive development mode. Some enterprises did not realize the importance of R\&D and innovation. The provincial and city governments nurtured SECLGs mainly by creating a sound development environment, providing essential production factors, and guiding enterprises to focus on $R \& D$ and innovation.

(1) Introduction and training of industrial talents

Talent advantage constitutes an important support for the growth and expansion of the SECLGs in Jiangsu Province, especially in southern Jiangsu Province. At the provincial level, the General Office of Jiangsu Provincial Party Committee and the provincial government released Opinions on Implementing 333 High-level Talents Cultivation Projects in Jiangsu Province in 2006 [6] and proposed that 30 middle-aged and young chief executive scientists, 300 young and middle-aged leaders, and 3000 young and middle-aged science and technology leaders should be selected from 2006. Through training, by 2010, among these high-level talents, there will be 10 academicians of the Chinese Academy of Sciences and Chinese Academy of Engineering; 150 distinguished scientists, engineering experts, and technological entrepreneurs; and 1500 science and technology leaders in all fields. At the municipal level, Suzhou introduced a series of policies in 2010, including the Implementing Rules of the Suzhou Innovation and Entrepreneurship Leading Talents Program, the Implementation Rules of Suzhou Key Industry Talents Project, the Implementing Rules of Suzhou Highly Skilled Talents Training Plan, and Opinions of Suzhou Municipality on Accelerating the Implementation of Overseas High-level Talents Introduction Project.

(2) Innovation of science and technology fund management

In 2008, the Jiangsu Provincial Party Committee and Provincial Government promulgated the Decision on Accelerating the Transformation of Economic Development Mode and clearly proposed to guide independent innovation and to substantially increase the contribution rate of science and technology to economic growth. Subsequently, the Jiangsu Provincial Department of Finance and Office of Science and Technology issued Measures for the Administration of Special Guiding Funds for Provincial Science and Technology Innovation and Achievements Conversion in Jiangsu Province, setting up special guiding funds for the transformation of major scientific and technological achievements and the construction of science and technology service platforms. In 2012, Jiangsu Province promulgated the Opinions of the Provincial Government on Accelerating the Combination of Science, Technology, and Finance and explored the establishment of a multi-level capital market system for science and technology. Suzhou released the Administrative Measures on the Use of Discounted Funds for Scientific and Technological Loans in Suzhou City in 2008 and the Provisional Measures for the Financing of Listed Scientific and Technological Enterprises in Suzhou City, which helped to finance science and technology enterprises through financial discounts and subsidized listings.

(3) Promotion of R\&D institutions

In 2006, Jiangsu Province promulgated the Notice of the Provincial Government on Encouraging and Promoting S\&T Innovation and Entrepreneurship, proposing to support the establishment of R\&D institutions in enterprises, to promote the development of R\&D institutions, and to support the establishment of R\&D institutions with foreign investment. The document stipulates that salaries for scientific and technical personnel at provincial-level technical centers, engineering centers, and engineering technology research centers should be deducted from the calculation of taxable income. Urban land use tax and property tax of the state-approved restructured scientific research institutions should be exempted from enterprise income tax within 5 years from the date of conversion. Foreign R\&D institutions that purchase patents, proprietary technology, and other expenses can apply for tax relief to tax authorities if they meet certain conditions. Suzhou released the Trial Measures on Encouraging the Establishment of R\&D Institutions in Suzhou Industrial Park in 2006 and proposed tax incentives and financial support for R\&D institutions set up in the park. In 2006, Zhenjiang introduced the Implementation Opinions of Zhenjiang Enterprises on Independent Innovation Projects, noting that it is necessary to enhance the core competitiveness of enterprises, to solicit business needs, and to enhance the service capability of research institutions.

\subsection{Second stage: systematically promote the development of small giant businesses}

At this stage, the majority of SMEs in southern Jiangsu gradually formed a consensus on technological innovation and intensive development. Many enterprises embarked on the road of "specialized, elaborative, and characteristic" development, and the conditions for the government to concentrate and guide the cultivation of small giant enterprises were gradually maturing. At the provincial level, the high-level design of cultivating "small specialists" was strengthened, and corresponding standards were formulated and improved. At the prefectural level, cities actively introduced the implementation policies and supporting policies. Provinces and municipalities formed a systematic effort to promote the cultivation of SECLGs, and one notable feature of this stage was that policies began to focus on the use of market 
mechanisms and mobilized all social forces in the form of government industry-led funds to support SECLG development.

(1) Building a system of nurturing policies

Since 2012, the Jiangsu Economic and Information Technology Commission has formulated the Special Instructions on Cultivating 10000 SECLG Enterprises in Jiangsu Province [5], which created a high-level policy design. After 2013, to guide the majority of SMEs in taking the road of "specialized, elaborative, and characteristic" development and in speeding up the cultivation of a group of small giant enterprises with strong scientific and technological innovation capability, high market share, and good quality and efficiency, Jiangsu started to annually appraise 50 "small technology giant" enterprises and 50 "special new products." As of the end of 2016, a total of 450 companies have been certification, of which 216 enterprises are located in southern Jiangsu. In 2015, Jiangsu introduced the Made in China 2025 Jiangsu Action Plan and clearly proposed that the province should develop a number of SECLG enterprises with prominent main businesses, strong competitiveness, and high growth potential and that it should focus on their market segments. Among southern Jiangsu Province cities, they developed the city's action plans and established the standards for municipal "small technology giant" enterprises and "special new products" to implement the planning goals at the provincial level.

(2) Supporting all kinds of entrepreneurship and innovation

Jiangsu has long recognized the importance of entrepreneurship and innovation. As early as 2006, Jiangsu promulgated the Opinions on Strengthening the Construction of High-level Entrepreneurship and Innovative Talents and led elites in promoting entrepreneurship and innovation. In 2015, Jiangsu expanded its support for entrepreneurship and innovation and released the Space Creation Plan for Promoting Public Innovation and Entrepreneurship (2015-2020), the Work Plan for Promoting Mass Creation in Jiangsu Province, and other relevant policies to encourage the participation of universities; research institutes; overseas high-level talents; science, technology, and management personnel; and other talents in innovation and entrepreneurship activities and to give support for high-level personnel in the fields of medical insurance, spousal employment, child education, housing, and so on. Jiangsu Province has set up a provincial award for outstanding contributions in science and technology. At the level of southern Jiangsu cities, Wuxi awards a maximum of RMB 350000 to science and technology innovation projects carried out by science and technology SMEs, and Zhenjiang awards RMB 500000 for each major achievement of independent innovation.

(3) Systematically integrating social resources

In 2010, Jiangsu formulated the Interim Measures for the Star Rating of SMEs' Public Service Platform in Jiangsu Province to standardize and guide the development of public service platforms for SMEs. In 2015, Jiangsu released the Notice on Carrying Out the Verification and Review of SMEs' Public Service
Star Platforms in 2015 to further standardize the construction of public service platforms for SMEs. To guide social capital in order to serve SMEs and make use of the capital market, Jiangsu set up the "specialized, elaborative, and characteristic board" in the provincial equity trading center and successfully landed 100 local enterprises. At the city level, the local governments attach great importance to the construction of public service platforms for SMEs. Their platform services cover many aspects, such as contractual intelligence audits, industrial design, information consultation, financial services, inspection, and testing. Suzhou issued a new Guidance and Evaluation Index System for SMEs in Suzhou City (Trial Implementation), established the SECLG list and classified guidance, summed up successful experiences, provided financing, technology, management, and other advisory services, and has regularly held SECLG policy interpretation and banking business docking activities. Nanjing set up an industry and information investment fund, which is mainly used to guide venture capital funds and other social capital to support start-up science and technology enterprises.

Some provincial and city supporting policies are shown in Table 2.

\section{SECLG nurturing strategy from the experience of southern Jiangsu Province}

At present, China is in need of a large number of SECLG enterprises to carry out strategies to create "manufacturing power" and a "strong industrial base." However, the cultivation of SECLG enterprises cannot be achieved in a single step. It requires the government to continually exert effort and gradually promote and formulate a good policy system, to provide a favorable environment, and to establish a sound long-term mechanism. The experience of southern Jiangsu Province may provide a reference for the whole nation with respect to aspects such as the market environment, service platform, entrepreneurship, innovation, leadership through demonstration, talent team building, and financial support. Such efforts will be beneficial for the construction of an echelon of SECLGs at the national, provincial, and municipal levels and will support the breakthrough of "manufacturing power" and "strong industrial base" strategies.

\subsection{Increasing government support}

Cultivating SECLGs requires that the government devote more attention to the development of SMEs and to consider the cultivation of SECLGs to be equally important as the nurturing of leading enterprises. We can refer to the experience of southern Jiangsu to establish SECLG Enterprise Libraries, provide classified guidance to such enterprises, provide consulting services regarding financing, technology, and management, and periodically organize policy interpretation. The demand for talent, capital, technology, and other special needs of SECLGs 
Table 2. Supporting policies of Jiangsu Province and southern Jiangsu cities.

\begin{tabular}{|c|c|c|}
\hline $\begin{array}{l}\text { Provincial } \\
\text { policies }\end{array}$ & \multicolumn{2}{|c|}{$\begin{array}{l}\text { Opinions on Implementing } 333 \text { High-level Talents Cultivation Projects in Jiangsu Province, } 2006 \\
\text { Opinions on Strengthening the Construction of High-level Entrepreneurship and Innovative Talents, } 2006 \\
\text { Notice of the Provincial Government on Encouraging and Promoting S\&T Innovation and Entrepreneurship, } 2006 \\
\text { Interim Measures for the Star Rating of SMEs'Public Service Platform in Jiangsu Province, } 2010 \\
\text { Opinions on the Implementation of the S\&T Entrepreneurship Project in Jiangsu Province, } 2010 \\
\text { Opinions of the Provincial Government on Accelerating the Combination of Science, Technology, and Finance, } 2012 \\
\text { Special Instructions on Cultivating } 10000 \text { SECLG Enterprises in Jiangsu Province, } 2012 \\
\text { Opinions on the Implementation of a National Innovation Zone in southern Jiangsu Province, } 2015 \\
\text { Notice on Carrying Out the Verification and Review of SMEs'Public Service Star Platforms, } 2015 \\
\text { Space Creation Plan for Promoting Public Innovation and Entrepreneurship, } 2015 \\
\text { Work Plan for Promoting Mass Creation in Jiangsu Province, } 2015 \\
\text { Preparatory Notice on Participation in the Opening Ceremony of the "Specialized, Elaborative, and Characteristic Board," } 2016\end{array}$} \\
\hline \multirow[t]{5}{*}{ City policies } & Nanjing & $\begin{array}{l}\text { Scientific and Technological Entrepreneurship Training Plan, } 2011 \\
\text { Implementation Plan for the Introduction of Leading Science and Technology Talents, } 2011 \\
\text { Nanjing SECLG Identification Measures, } 2014 \\
\text { Nanjing SME Public Service Platform Identification Measures, } 2012 \\
\text { Nanjing Science and Technology Bank Innovation and Development Implementation Measures, } 2015\end{array}$ \\
\hline & Suzhou & $\begin{array}{l}\text { Trial Measures on Encouraging the Establishment of R\&D Institutions in Suzhou Industrial Park, } 2006 \\
\text { Measures for the Management of Special Funds for Credit Risk Compensation of SMEs in Science and Technology } \\
\text { in Suzhou City, } 2009 \\
\text { Suzhou Finance Supporting Enterprise Independent Innovation Action, } 2015 \\
\text { Suzhou "Special New Products" Identification Measures, } 2013 \\
\text { Guidance and Evaluation Index System for SMEs in Suzhou City (Trial Implementation), } 2016 \\
\text { Suzhou Science and Technology Service Organization Registration and Performance Management Measures, } 2016\end{array}$ \\
\hline & Wuxi & $\begin{array}{l}\text { Regulations for Promoting the Restructuring of SMEs in Wuxi, } 2014 \\
\text { Wuxi "Special New Products" Identification Measures, } 2015 \\
\text { Opinions on Strengthening the Construction of Skilled Talent Team and Promoting Industrial Transformation and } \\
\text { Upgrading, } 2015\end{array}$ \\
\hline & Changzhou & $\begin{array}{l}\text { Changzhou Science and Technology Incentive Measure, } 2011 \\
\text { Implementation Measures on Promoting the Construction of Company R\&D Institutions in Changzhou, } 2012 \\
\text { Science and Technology SME Identification Rules of Changzhou, } 2013 \\
\text { Management Measures of the Changzhou Leading Talent Enterprise Microfinance Guarantee Insurance Special } \\
\text { Fund, } 2015\end{array}$ \\
\hline & Zhenjiang & $\begin{array}{l}\text { Implementation Opinions of Zhenjiang Enterprises on Independent Innovation Projects, } 2006 \\
\text { Zhenjiang Municipal Science and Technology Innovation Special Fund Management Measures, } 2013 \\
\text { Zhenjiang Science and Technology Award Measures, } 2015 \\
\text { Zhenjiang "Special New Products" Identification Measures, } 2016\end{array}$ \\
\hline
\end{tabular}

is urgent. The government needs to eliminate obstacles in various institutional mechanisms that limit the free flow of capital, technology, property rights, talents, and labor and that promote production factors in accordance with the laws of the market. We must pay special attention to improving the non-discriminatory and quality-enhancing procurement policies in the government and leading state-owned enterprises so as to truly protect innovation-driven suppliers and to promote survival of the fittest. We need to optimize the development environment and eliminate market barriers for SECLG enterprises.

\subsection{Building a linked policy system among national, provincial, and municipal levels}

In 2012, Jiangsu issued Special Instructions on Cultivating 10000 SECLG Enterprises in Jiangsu Province as a general outline. Under such guidance, cities of Southern Jiangsu have actively promulgated local implementation plans and standards and formed a provincial, municipal linkage in the SECLG cultivation system. At present, in the cultivation of SECLGs at the national level, a cultivation system closely coordinated by prov- inces and municipalities can also be formed under the guidance of the Implementation Plan of Cultivating and Upgrading Single Champions in the Manufacturing Industry. We need to nurture SECLGs by adhering to the combinations of enterprise leadership and government guidance, cultivation and promotion, and demonstration and popularization; by establishing a national, provincial, and municipal SECLG echelon, and by constantly improving the standards in order to create a series of successful experiences and a development model to encourage more companies to take the "specialized, elaborative, and characteristic" single-champion development path.

\subsection{Strengthening the industrial chain, innovation chain, capital chain, and policy chain interaction}

We need to establish a mechanism that integrates all social forces, strengthens the interaction between the industrial chain, innovation chain, capital chain, and policy chain, and jointly supports the development of SECLG enterprises. First, it is important to promote cooperation among industries, universities, and research institutes and take measures to support SMEs in 
conducting product R\&D and technical research and transforming the obtained results. Second, it is necessary to strengthen the cooperation of SECLG enterprises with leading enterprises, especially with respect to pushing SECLGs into the supply chain of leading enterprises and forming a long-term cooperation mechanism. Third, it is necessary to speed up the construction of cooperative supporting platforms for SMEs and to organize coordination and supporting activities in order to gradually improve cooperation between SMEs. Fourth, it is important to guide social capital to serve SECLG enterprises, for instance, by establishing a "specialized, elaborative, and characteristic board" in the capital market and forming industrial and information industry investment funds to guide social capital into science and technology start-ups in key areas. Fifth, it is essential to improve the mechanism of excavation, selection, cultivation, maturity, and exit of SECLG enterprises through the annual selection of "national single champion cultivation enterprises and demonstration enterprises" and to support breakthroughs in the key industrial base areas.

4.4 Fostering the publicity of the "specialized, elaborative, and characteristic" industrial culture at the national level

It is very important to make promotion efforts at the national level in order to nurture the SECLG enterprises. First, with reference to the experience of southern Jiangsu Province, case analysis of single champion enterprises needs to be conducted at the national level; their modes of and experience with innovation and development, personnel training, development paths, business philosophies, and product plans need to be summarized; and such findings need to be published for all of society. Second, it is important to promote the "specialized, elaborative, and characteristic" culture and the spirits of innovation, integrity, and craftsmanship in order to guide massive SMEs in taking the path of specialized, elaborative, and characteristic development. Third, it is important to carry out various types of exhibition activities to promote industrial culture on a regular basis in order to focus society's attention on the development of SECLG enterprises. Special live meetings may be held to exchange experiences with nurturing SECLGs and forming a supporting atmosphere for SECLGs to participate in the "strong industrial base strategy."

\section{Conclusions}

SMEs have a high degree of complexity and have different growth paths. Therefore, the ways and means for nurturing SECLGs in different regions vary. Special research to create a good market environment, provide strong policy support, and effectively support SECLG enterprises to overcome difficulties, develop breakthroughs in key manufacturing areas, and promote China's manufacturing sector to step into the mid-to-high-end fields will always have theoretical and practical significance. Based on the achievements of the Consulted Projects of the China Academy of Engineering "Manufacturing Power Strategy" and "Industrial Strong Base Strategy", this paper takes the example of SECLG enterprises in southern Jiangsu Province to systematically analyze local measures of cultivation, to summarize the related experience and characteristics, and finally to propose ideas and suggestions on how to promote the cultivation of SECLG enterprises nationwide. Combined with the practice of the "Made in China 2025 Pilot Demonstration Zone" in southern Jiangsu, we can further analyze the relationship between the cultivation policies and cultivation results of the SECLGs in southern Jiangsu Province, strengthen research on the internal mechanism of interaction among the industrial chain, innovation chain, capital chain, and policy chain, and provide more in-depth study of an "industrial strong base."

\section{References}

[1] Simon H. Invisible champion: The world's best 500 companies success way [M]. Beijing: The Economic Daily Press, 2005. Chinese.

[2] Li T S. Basic characteristics and nurturing mechanisms of "specialized, elaborative, characteristic and innovative" SMEs-Take Shanghai as an example [J]. Special Zone Economy, 2012 (7): 67-69. Chinese.

[3] Sun C L. Cultivate more science and technology "little giant" enterprises [J]. China Small \& Medium Enterprises, 2013 (1): 14 Chinese.

[4] Zhao H C. Suggestions on the upgrade path of science and technology "little giant" enterprises in Tianjin [J]. Tianjin Economy, 2016 (5): 70-73. Chinese.

[5] Jiangsu Economic and Information Technology Commission. Guidelines of the nurturing of ten thousand "specialized, elaborative, characteristic and innovative" SMEs in Jiangsu Province [EB/ OL]. (2012-02-20) [2017-04-23]. http://www.xzqs.gov.cn/Article/ Show?id $=40602$. Chinese.

[6] Jiangsu Provincial Party Committee Office, Jiangsu Provincial Government Office. Opinions on the implementation of the "333 high-level personnel nurturing project" in Jiangsu Province [EB/ OL]. (2006-07-26) [2017-04-26]. http://www.rccs.gov.cn/content/ article.asp?typeid $=10 \& \mathrm{id}=8$. Chinese. 